\title{
X: Aniversario de la Sociedad Peruana de Historia
}

La "Sociedad Peruana de Historia", activa y prestigiosa institución nacional de historiadores profesionales ha celebrado, el día 12 de julio del año presente, el $\mathrm{X}^{\text {? }}$ aniversario de su declaración de principios, contenida en el Acta de Fundación.

Rige la "Sociedad Peruana de Historia" una Directiva, constituida por el Dr. Carlos Daniel Valcárcel: Director, Dr. Carlos Radicati di Primeglio: Secretario General, Prof. Ricardo Arbulú Vargas: Secretario de Aclas, Dr. Alberto Tauro: Secretario de la Revista, Dr. Raúl Rivera Serna: Bibliotecario-Archivero, y Prof. Alejandro Hernández Robledo: Tesorero. Han sido Directores de la SPH, 1 a Dra. Ella Dunbar Temple y el Dr. Pedro Benvenuto Murrieta, respectivamente. Los restantes Miembros del Número, por Corden Cdel antigüedad, osn: Dr. Gustavo Pons MuzzoJoDr.e Teodoro MenesesjePso, Guillermo Lohmann Villena, Dr. Javier Pulgar Vidal, Dr. Jorge C. Muelle, Dr. Alberto Santibáñez Salcedo, Dr. Vicente Ugarte del Pino, Dr. Bolívar Ulloa, Dr. Juan B. Lastres, Dr. José M. Vélez Picasso. Como se ve, la mayor parte son Catedráticos de la Universidad de San Marcos. Oiros de la Universidad Católica, de la Biblioteca Nacional y de instituclones rectoras de la cultura peruana. Hay Miembros Correspondientes nacionales y extranjeros. Nacionales: Dr. Horacio Villanueva Urteaga (Cusco), Dr. Efraín Morote Best (Cusco), Dr. Vladimiro Bermejo (Arequipa). Dr. Washington Cano (Puno), y Dr. Alberto Casa Vilca (Ica). Extranjeros: en Alemania, Argentina, Chile, Ecuador, España, Estados Unidos de Norteamérica, Francia, México y Puerto Rico.

La Sociedad fué oficialmente reconocida el 13 de octubre de 1945. por Resoluctón Suprema No 3279. El Estatuto se aprobó el año 1945; el Reglamento, en 1946. El Escudo de la SPH ha sido publicado en el tomo I de su revista Documenta. Hasta el presente ha realizado más de 208 Sesiones académicas y administrativas, disertando los Miembros de 
Número, los Miembros Cornespondientes - peruanos y extranjerose invitados especiales. Ha estado representada en las principales reuniones internacionales y del país.

Sus publicaciones comprenden dos grupos: I) Biblioleca, que hasta el presente consta de dos Series y cinco Volúmenes. A) Serie Monografías: 1. Usca Paucar, drama quechua del siglo XVIII, por Teodoro. L. Meneses. 2. Crónicas Perdidas, Presuntas y Olvidadas sobre la Conquista del Perú, por Raúl Porras Barrenechea. 3. Introducción al estudio de los Quipus, por Carlos Radicati di Primeglio. 4. Ignacio de Castro, humanista tacneño y gran cusqueñista (1732-92), por Daniel Valcárcel. B) Serie Registro Hístórico (documentos): Libro de Oposiciones de la Universidad de San Antonio del Cusco (siglo XVIII), pub. por Daniel Valcárcel. II) Hemeroteca, que consta de 3 tomos: tomo I, 1948, 593 páginas; tomo II, 1949-50, 560 páginas; tomo III, 1951-55, 826 páginas; tomo IV, en preparación. El tomo inicial de la nueva Serie Bibliografía aparecerá a fines del próximo año.

Caracierística saltante de la Sociedad Peruana de Historia es la de constituir un grupo de trabajo peruanista, donde la tarea de coniunto orienta el esfuerzo de cada uno de sus miembros. A través del trabajo individual, persíguese el objetivo genérico de descubrir el sentido orgánico de la cultura peruana.

\section{Biblioteca de Letras "Jorge Puccinelli Converso»}

